\title{
Corela
}

Cognition, représentation, langage

12-1 | 2014

Vol. $12, \mathrm{n}^{\circ} 1$

\section{Vers une analyse des marqueurs yet et still : There is still much to say}

\section{Lise Hamelin}

\section{OpenEdition}

\section{Journals}

Édition électronique

URL : http://journals.openedition.org/corela/3439

DOI : 10.4000/corela.3439

ISSN : $1638-573 \mathrm{X}$

\section{Éditeur}

Cercle linguistique du Centre et de l'Ouest - CerLICO

\section{Référence électronique}

Lise Hamelin, "Vers une analyse des marqueurs yet et still : There is still much to say », Corela [En ligne], 12-1 | 2014, mis en ligne le 25 juin 2014, consulté le 05 mai 2019. URL : http:// journals.openedition.org/corela/3439; DOI : 10.4000/corela.3439

Ce document a été généré automatiquement le 5 mai 2019.

\section{(c) (i) (2)(2)}

Corela - cognition, représentation, langage est mis à disposition selon les termes de la licence Creative Commons Attribution - Pas d'Utilisation Commerciale - Partage dans les Mêmes Conditions 4.0 International. 


\title{
Vers une analyse des marqueurs yet et still: There is still much to say
}

\author{
Lise Hamelin
}

1 Dans cet article, nous proposons de rendre compte du fonctionnement des marqueurs de l'anglais contemporain yet et still. Plus particulièrement, à travers l'examen de leurs contextes d'apparition et des contraintes qui régissent leur emploi, on s'efforcera de proposer une analyse unitaire de leur modus operandi en faisant émerger les opérations qui leur sont associées et en mettant en évidence les raisons de leur possible concurrence dans des configurations bien particulières.

2 Rappelons très brièvement et schématiquement que yet et still sont deux marqueurs de nature adverbiale qui peuvent apparaître dans des contextes dans lesquels ils sont associés à une valeur que l'on peut qualifier de temporelle ou des contextes dans lesquels ils reçoivent une valeur concessive.

\section{Yet et la quantification : yet more, yet another}

3 Yet est un marqueur qui apparaît typiquement dans des contextes non assertifs ou assertifs négatifs. Ceci est un fait bien connu, mentionné dans les grammaires comme principe de fonctionnement du marqueur (Swan 1980, \$579; Quirk et alii. 1985, §10.60). On peut noter toutefois que celui-ci est, en fait, susceptible d'apparaître en contexte assertif positif sous des conditions bien particulières, comme en (1) à (3) :

(1) I'm so pleased they haven't put the cottage on the market because that would leave it empty. Yet another empty cottage here would be just dreadful. (British National Corpus) (2) As Jung said, an attempt to leave behind the child which still lives in us all, denies a part of ourselves and results in behaviour of a very childish, as opposed to childlike, kind. What happens when the unconscious child in Joan Halton finds yet again that she is always losing out, this time to her husband, to his old car on which he dotes or to his mother who is still demanding so much of his time and attention, and that he appears to be forgetting that he is now married and has new first loyalties? (BNC)

(3) French troops, deafened by the thunderous roar, felt yet more deaf with the unexpected, but long-prayed for, silence. (BNC) 
Tous ces exemples contiennent un terme renvoyant explicitement à de la quantification, que ce soit la mention du degré en (3) avec more deaf, ou encore d'une occurrence supplémentaire : un individu, en (1) avec another empty cottage, ou un événement, en (2) avec again. On pourra proposer des gloses faisant apparaître le marqueur once en (2'), ou bien even lorsque yet porte sur un degré de propriété, comme en (3'), par exemple :

(2') [...] What happens when the unconscious child in Joan Halton finds once again that she is always losing out [...]

(3') French troops, deafened by the thunderous roar, felt even more deaf with the unexpected, but long-prayed for, silence.

Ce n'est pas yet seul qui signale que l'on ajoute un degré supplémentaire, ou une occurrence supplémentaire, mais les termes again, another ou more, avec lesquels il fonctionne. Cependant, il semble qu'on ne puisse pas employer yet dans un contexte assertif positif en l'absence de tels termes. Ainsi, les énoncés (1') à (3"), dans lesquels ils ont été supprimés, sont inacceptables :

(1') ? I'm so pleased they haven't put the cottage on the market because that would leave it empty. Yet an empty cottage here would be just dreadful.

$(2 ") *[. .$.$] What happens when the unconscious child in Joan Halton finds yet that$ she is always losing out [...]

$(3 ") *$ French troops, deafened by the thunderous roar, felt yet deaf with the unexpected, but long-prayed for, silence.

6 La présente analyse s'inscrit dans le cadre de la Théorie des Opérations Enonciatives d'Antoine Culioli. Elle propose de considérer que yet et still portent sur des occurrences de notions qui peuvent être nominales ou verbales. Les occurrences sont des individus discursifs qui constituent des modes d'appréhension d'une notion. Celles-ci sont construites par deux opérations de prélèvement sur la notion :

- un prélèvement d'abord quantitatif par lequel on met en place un individu occupant une portion d'espace-temps n'ayant d'autres propriétés que celles que lui confère son appartenance à la notion,

- puis, un prélèvement qualitatif fondé sur le fait que l'occurrence ainsi construite est susceptible d'acquérir des propriétés qui lui appartiennent en propre et la rendent particulière, distinguable de toute occurrence de la notion (cf. Culioli, 1999, pp. 10-11).

7 Si ces deux opérations sont parvenues à leur terme, l'occurrence est dite discrète et stabilisée quantitativement et qualitativement, c'est-à-dire qu'elle occupe une portion d'espace-temps, d'une part, et qu'elle possède des propriétés singularisantes, d'autre part. Si l'occurrence ne possède pas de propriétés de cet ordre, et qu'elle constitue simplement un exemplaire quelconque de la notion qui ne se définit que par l'espacetemps qu'elle occupe, elle est dite dense. Lorsque l'occurrence constitue un renvoi direct à la notion, lorsqu'elle ne possède aucune propriété singularisante ni n'occupe aucun espace-temps délimité, elle est dite compacte.

Another, more, ou again sont des marqueurs qui appartiennent à des catégories grammaticales différentes et ont donc des fonctionnements syntaxiques distincts, mais ils ont en commun le fait qu'ils peuvent construire des occurrences discrètes de la notion sur laquelle ils portent. Another indique, en effet, qu'on se trouve en présence d'une occurrence supplémentaire, distinguable qualitativement (parce qu'elle possède des propriétés spécifiques) et quantitativement (parce qu'elle occupe une portion d'espacetemps particulière) des occurrences déjà construites. Again joue un rôle similaire, à ceci près que l'occurrence en question n'est alors pas nominale, mais verbale. Quant à more, il 
permet la construction d'un degré supplémentaire d'une propriété particulière. Ce degré peut être perçu comme un au-delà et être qualitativement distinguable du préconstruit, ou s'inscrire dans une gradation construite comme un continuum, dans ce cas-là, il n'y a pas d'opposition qualitative possible. Tout dépend du type de propriété associé à more et des implications sémantiques et pragmatiques de l'énoncé. Yet s'associe donc, dans chaque cas, à un contexte élaborant ou pouvant élaborer un ensemble d'occurrences $\mathrm{N}+1$, c'est-à-dire posant l'existence d'une occurrence supplémentaire, ou d'un degré autre, par rapport à ce qui est déjà construit dans le contexte.

Dans les énoncés de cet ordre, le contexte suggère souvent que le degré maximal ou que le nombre maximal d'occurrences avait été atteint. Le lecteur pourra observer les passages que nous avons soulignés dans les exemples (1) à (3), ou s'attarder sur (4) à (6) ci-dessous :

(4) But if Creggan's own sense of imprisonment was made worse by the sight of other caged creatures, it was heightened yet further by the fact that small song birds like sparrows, thrushes and starlings would dart freely in between the bars of the Cages and take advantage of what they found there. (BNC)

(5) At first there was reluctance to jeopardise the concessions already gained by pushing for yet another. (BNC)

(6) The content and implications of this document have been very widely analysed and debated as has the Tanzanian reaction to it, but a few short passages need perhaps to be quoted yet again if only to underline the very fundamental challenges it poses for curriculum planners. (BNC)

10 Les passages soulignés dans ces trois exemples indiquent que l'occurrence supplémentaire ou le degré supplémentaire dénoté par further, another ou again n'était $a$ priori pas attendu, ne correspondait pas aux représentations du sujet-point de vue dominant l'énoncé. Cela est construit en (4) par la locution concessive But if à l'initiale de l'énoncé, qui indique que les choses auraient dû en rester là, par there was reluctance en (5), qui met en cause la justification de l'existence d'une occurrence supplémentaire de concessions, et en (6), par The content and implications of this document have been very widely analysed and debated, qui semble présupposer que a few short passages need to be quoted devrait ne pas être le cas, ce qui est renforcé par la présence dans l'énoncé des marqueurs but et perhaps. Pourtant, dans chaque cas, l'énonciateur fait le constat de l'existence en situation de cette occurrence que l'on pourrait, par conséquent, qualifier d'excédentaire.

11 Comme on l'a montré, cette nuance sémantique est construite par la présence dans l'énoncé de termes véhiculant une forme de valuation de l'occurrence sur laquelle porte yet. Toutefois, celui-ci vient renforcer, appuyer l'idée selon laquelle cette occurrence représente un au-delà quantitativement et qualitativement distinct des occurrences précédentes. On peut le constater dans certains énoncés, comme (7), dans lequel la présence de yet suggère que, finalement, on aurait pu arrêter là l'énumération d'exemples, la démonstration aurait tout de même été convaincante :

(7) She handed him the ring, telling him never to part with it. When Bassanio asked the doctor, a dressed up Portia, how he could pay her, she answered that she would take nothing but the ring. He refuses to give it, but after much persuasion from Antonio, he decides to give it to her. This testing of bassanio [sic], her own husband who she is meant to love and trust, tells us how cunning, powerful and suspicious she really is. Her attitude towards all the different suitors is yet another example of her immorality and dishonesty. In Act 1 Scene 2, between lines 33-91, she is dictated a list of suitors to which she is both critical and particular. When Bassanio 'a Venetian, a scholar and a soldier' is mentioned, in Act 1 Scene 2 lines 
92-94, she replies (line 95) : Yes, yes it was Bassanio! -- as I think so he was called.

His name had not been mentioned to her at all [...] (BNC)

12 Yet, systématiquement associé à des occurrences quantitativement et qualitativement individuées, met l'accent sur le caractère spécifique de l'occurrence sur laquelle il porte, lui conférant ainsi un statut hautement singulier, presque remarquable. On observe donc que yet permet, en contexte assertif positif, l'introduction d'une occurrence ou d'un degré supplémentaire, inattendu, autrement dit, renforce le caractère singulier de cette occurrence par comparaison avec les occurrences de la même notion déjà construites en amont.

13 Par conséquent, on formulera l'hypothèse selon laquelle yet serait la trace d'une opération de disjonction portant sur les délimitations quantitative et qualitative de l'occurrence. Celle-ci occupe un espace-temps autre et possède des propriétés distinctes par rapport aux occurrences déjà construites.

Still, régulièrement envisagé comme le pendant positif de yet, peut être comparé à ce marqueur dans la mesure où il est, lui aussi, susceptible d'être associé à la mention d'un degré, comme en (8).

(8)It is important to win, but still more important to fight. (BNC)

Ou encore en (9), dans lequel still peut être glosé par even (9') ou par yet (9").

(9)He snarled again and lifted his paw still higher. (BNC)

(9') He snarled again and lifted his paw even higher.

(9") He snarled again and lifted his paw yet higher.

15 Dans les exemples de cet ordre, still est généralement suivi de more, que ce dernier porte sur un adjectif, et donc, sur un degré de propriété comme en (8) et (9), ou détermine un substantif, comme en (10):

(10) Mrs June Goodier, one of the organisers, said they were delighted with the amount raised with still more money to come in. (BNC)

Still et yet sont donc tous deux susceptibles d'apparaitre dans ce type de contextes. On note, toutefois, une légère différence de sens, l'énoncé (11) impliquant en fait «one may think enough money had been raised ", ce qu'on n'a pas avec still en (11') :

(11) The next step is to raise yet more money to renovate the other side. (BNC)

(11') The next step is to raise still more money to renovate the other side.

17 En effet, en dépit des similitudes observées, yet et still, ont un fonctionnement différent dans ce type d'énoncés. Ainsi, on trouvera (12), par exemple, mais pas (12') :

(12) Times being hard, the vicious squabbles that have plagued Peru since the 1960s have broken out again, between raw-material exporters (who want freer trade) and manufacturers (who want still more protection). (BNC)

$(12 ') *$ Times being hard, the vicious squabbles that have plagued Peru since the 1960s have broken out again, between raw-material exporters (who want freer trade) and manufacturers (who want yet more protection).

18 Protection est un nom dont le fonctionnement est dense. Le contexte exprime, par ailleurs, l'idée de la non-interruption du désir ou du besoin des référents de manufacturers, via le verbe want. More protection est donc construit ici dans la continuité de l'existant, et non comme un individu singularisé, supplémentaire, s'ajoutant potentiellement à ce dont l'existence a déjà été mise en place.

19 Ce sont des arguments similaires qui peuvent expliquer que, alors qu'en (9) comme en (10), le fonctionnement de still est assez proche de celui de yet, on ne pourrait le substituer à ce marqueur en (1) et (2) : 
(1'") ? I'm so pleased they haven't put the cottage on the market because that would leave it empty. Still another empty cottage here would be just dreadful.

$\left(2^{\prime \prime \prime}\right) *$ I note still again the Labour party's hostility to any persons exercising any choice in the interests of their family.

En (1'"), l'interprétation de still, à condition que celui-ci soit suivi d'une pause intonative, ne pourrait être que concessive (in spite of that, nonetheless, etc.), ce qui n'est pas cohérent dans le contexte de l'énoncé. Quant à (2"'), il est tout simplement inacceptable.

En (9) et (9"), avec He snarled again and lifted his paw still/yet higher, on a vu que yet était substituable à still. L'énonciateur met alors en place un jeu sur le gradient associé à la notion de propriété dénotée par high. Autrement dit, on mesure le degré auquel le référent de his paw possède la propriété dénotée par high par comparaison avec un état antérieur, une position de référence. En fait, en fonction du point de vue adopté, on peut considérer :

- soit que l'on met en regard deux points distincts du gradient, un degré supérieur et un degré inférieur, qui se présentent comme des échelons particuliers et s'inscrivent donc en rupture l'un par rapport à l'autre, c'est ce que fait yet en (9")

- soit que le passage d'un degré à l'autre s'inscrit dans un continuum, et qu'il n'y a pas de rupture nette, juste une progression, c'est ce qui se passe avec still en (9).

Une telle analyse est à même de rendre compte de l'impossibilité de substituer yet à still en (12), puisque cet énoncé implique l'existence d'une forme de continuité dans la réalisation de <manufacturers - want protection>. Elle permet aussi d'expliquer les problèmes engendrés par la substitution de still à yet en (1) et (2), qui sont des énoncés dans lesquels les occurrences sur lesquelles portent yet sont discrètes, et donc quantitativement et qualitativement individuées, ce qui fait obstacle à l'idée d'une progression ou d'une continuité que nous associons à still.

22 A la suite de cette remarque, on formulera l'hypothèse selon laquelle still, quant à lui, serait la trace d'une opération de différenciation portant sur les délimitations de l'occurrence à laquelle il est associé, dans la mesure où il inscrit cette occurrence dans une zone contiguë par rapport à ce qui a été construit en amont.

On note, par ailleurs, que seul still permet de parcourir le gradient dans les deux sens, du moins vers le plus, mais aussi du plus vers le moins :

(13) Thus the most autonomous of fields is the scientific field. The academic field and artistic field (high culture) occupy rather intermediate positions, autonomy in the former being from the field of power and in the latter from consumers in the social field. Still less autonomous is the legal field. (BNC)

$\left(13^{\prime}\right)$ * Thus the most autonomous of fields is the scientific field. The academic field and artistic field (high culture) occupy rather intermediate positions, autonomy in the former being from the field of power and in the latter from consumers in the social field. Yet less autonomous is the legal field.

24 Yet signale que l'occurrence supplémentaire ou le degré supplémentaire s'inscrit en altérité par rapport aux représentations véhiculées dans le co-texte gauche. Cette représentation correspond à l'idée selon laquelle la quantité maximale de $\mathrm{X}$ ou le degré maximal auquel $\mathrm{X}$ peut posséder la propriété $\mathrm{Y}$ a été atteint. De fait, l'altérité se conçoit nécessairement comme un au-delà, une occurrence dont nous avons dit qu'elle était excédentaire, et il ne saurait être question de retrancher une occurrence ou de reculer sur le gradient avec yet. 
On rencontre quelques rares énoncés qui semblent être des contre-exemples, dans lesquels yet est associé à less, mais en réalité, dans ces énoncés, il ne s'agit pas d'opposer deux degrés distincts de la même propriété, mais deux degrés de propriétés différentes. On ne se situe pas, en réalité, sur le même gradient, puisqu'on ne parle pas de la même notion de propriété. C'est le cas en (14), dans lequel on met en relation un certain degré de consciousness avec un certain degré (inférieur) de fastidiousness :

(Elsewhere, off the creek bank, children, mostly older boys, swam. There was not much consciousness of "polluted" waters in those days and yet less fastidiousness on the part of energetic farm boys.) (Corpus Of Contemporary American English)

Dans un énoncé comme (14), l'altérité entre le degré de propriété sur lequel porte yet et le degré de consciousness construit dans le co-texte gauche ne peut être éliminée, puisqu'il est question de propriétés distinctes l'une de l'autre.

Dans le même ordre d'idée yet peut être associé à l'expression d'une durée, comme en (15) et (16), dans lesquels some months et years sont présentés comme un temps additionnel, un délai ou un report inattendu :

(15) It could be some month yet before the pensioners know how much they'll get, and when they'll get it. (BNC)

(16) It was not a fortune, but it was a respectable competence, thanks to royalties, which would continue for years yet, whether the doctor reappeared or remained limbo. (BNC) Dans ces énoncés, still n'est pas acceptable :

$\left(15^{\prime}\right)$ ? It could be some month still before the pensioners know how much they'll get, and when they'll get it.

(16') ? It was not a fortune, but it was a respectable competence, thanks to royalties, which would continue for years still, whether the doctor reappeared or remained limbo.

Contrairement à yet, still semble marquer plutôt l'idée d'une continuité. C'est probablement la raison pour laquelle on ne le rencontre pas dans ce type de contextes, dans lesquels les termes some month ou years sont présentés comme des délais supplémentaires ou additionnels, alors même qu'il est parfaitement compatible avec l'expression d'une durée, comme on peut le voir en (17). De ce point de vue, la position de still, différente de cette de yet, ne nous parait pas anodine :

(17) Amy said, suddenly serious : 'I'll think of something. There's still four weeks to

go. [...] I promise you that libel action will never come to court.' (BNC)

Avec yet, le délai supplémentaire peut aussi être conforme à ce qui était attendu, comme en (18) :

(18) Just then Biff started clanging the iron triangle to summon them together. 'Bit early, isn't he ?' grumbled Sam.' There's ten minutes to go yet, I reckon.' (BNC)

Néanmoins, on peut noter que la mention de ten minutes to go est construite en opposition à ce qui apparaît dans le co-texte gauche, à savoir Biff started clanging the iron triangle to summon them together, qui suggère au contraire que le temps imparti est terminé. Encore une fois, la différence entre yet et still dans ce type de contexte semble être que still est incompatible avec toute forme de discontinuité, alors même que yet construit la durée restante comme étant au-delà de ce qui est construit comme attendu.

31 Yet renforcerait une relation d'altérité déjà existante entre le terme qui le suit et ce qui est construit dans le co-texte gauche, alors que still semblerait, lui, davantage compatible avec l'absence de rupture par rapport à ce qui précède. Cela explique leurs affinités, pour yet, avec des occurrences discrétisées, quantitativement et qualitativement individuées, et pour still, avec des occurrences au caractère dense, sans individualité qualitative. 


\section{Yet et still marqueurs de concession} relation <he - take to show business like a duck to water>, du fait des propriétés associées à / show-business/, est présentée comme incompatible a priori, ou au moins contradictoire, avec son appartenance à la classe dénotée par /a strict Baptist/. Si on manipule l'énoncé comme proposé en (19"), son acceptabilité est remise en question pour des raisons pragmatiques :

$\left(19^{\prime \prime) ~ ? ~ S t e v e ~ w a s ~ b r o u g h t ~ u p ~ a s ~ a ~ s t r i c t ~ B a p t i s t . ~ Y e t ~ h e ~ j o i n e d ~ h i s ~ l o c a l ~ T e m p e r a n c e ~}\right.$ Society. A la différence de <he - take to show business like a duck to water>, <he-join his local Temperance Society> est parfaitement cohérent avec les propriétés associées à la notion / Baptist/. Or, la présence de yet, dans cette manipulation, semble indiquer que ces deux relations sont considérées comme contradictoires par l'énonciateur. individués. En effet, dans le BNC, dans le co-texte droit ( 6 termes) de yet, le verbe lexical le plus fréquemment associé à la forme -ing, looking, n'apparaît qu'en 43ème position. Cela implique que l'ancrage situationnel de l'événement à droite du marqueur ne présente pas d'intérêt particulier avec yet. Bien sûr, la concession repose sur l'opposition de représentations, et donc, sur des considérations essentiellement qualitatives, ce phénomène n'est donc pas le seul fait de la présence de yet dans l'énoncé. Il est toutefois intéressant de noter que si still semble adopter un comportement assez similaire, certaines données quantitatives permettent de montrer qu'il en va autrement.

En effet, si on s'intéresse au co-texte droit de still dans ce type de contexte, on s'aperçoit que le terme given y est surreprésenté :

(20) Our job as music teachers is much broader than working with those few students who wish to follow in our footsteps. We must encourage and inspire all of the students involved in our programs. Still, given the powerful influence that this survey attributed to the high school music teacher, we must consider how we might identify and nurture potential teachers more directly. (COCA)

Dans cet exemple, il ne s'agit pas d'opposer des points de vue, mais d'introduire un nouvel élément permettant de nuancer dans la suite de l'énoncé les propos tenus en premier lieu. Il ne s'agit donc pas, comme en (21), dans lequel il serait difficile de 
substituer still à yet, de mettre en relation des représentations complètement contradictoires :

(21) He is capable of killing 10 men in a matter of seconds. Yet/ ? Still, he can also love. (COCA)

40 Un autre élément allant dans ce sens est la fréquence, derrière still d'énoncés que l'énonciateur ne prend pas réellement en charge. On trouve à foison des demandes de confirmation adressées au co-énonciateur comme en (22), des proverbes ou d'autres éléments relevant de la sagesse populaire comme en (23), ou encore des énoncés comme (24), dans lequel l'énonciateur repère la relation prédicative à droite de still par rapport à un sujet-point de vue universel, par le biais de la valeur générique associée au pronom we. En fait, tout se passe comme si, avec still, l'énonciateur s'inscrivait dans une démarche de recherche de consensus :

(22) 'You know, I wanted to take you to lunch at the Ritz, like we did last time, but it would have made us so late in Calking. Still, there'll be another chance, won't there? (BNC)

(23) It would have been better, however, if Seikosha had included at least one more as standard. Still you can't have it all. (Web : google.co.uk)

(24) Nor does leading a healthy life mean we must be vaccinated for every possible disease. Still, if we travel in an infected area, we would be foolish not to take precautions. (Web : google.co.uk)

41 Dans ces énoncés, l'énonciateur ne présente pas le contenu propositionnel introduit par still comme relevant de sa subjectivité propre, mais comme constituant une représentation pouvant être endossée par n'importe quel sujet point de vue. En ce sens, ces énoncés se voient associés à une sorte de prise en charge générique, ce qui rend impossible la construction d'une altérité forte entre les représentations véhiculées par le co-texte gauche et le co-texte droit. En effet, si ce qui apparaît à droite du marqueur est susceptible d'être pris en charge par n'importe quel énonciateur, alors cela ne peut s'inscrire en rupture avec les représentations construites à gauche du marqueur. C'est ainsi que l'on voit émerger systématiquement des phénomènes de recherche de consensus, d'apport d'une nuance par rapport à ce qui a été dit, en d'autres termes, l'idée qu'une opposition réelle, forte, entre les représentations construites à gauche et à droite du marqueur ne peut exister.

Les contextes de cet ordre ne sont pas strictement impossibles avec yet, mais ils sont nettement moins fréquents. Alors que yet est plus répandu que still dans son emploi de conjonction, on trouve dans google.co.uk 76 occurrences de "Still you can't have it all », contre 1 seule occurrence de "Yet you can't have it all »:

(25) The band have recently been trying to get fans to bring flags to their gigs quipping that "everyone needs a novelty, and we have plenty of them" but also to try and get fans to bring their own flags as it's something you can identify with. Sadly the gig only has one, very large, Croatian flag and a few small English flags teetering nervously on the end of sticks. Still, you can't have it all. (Web: google.co.uk)

(26) Perhaps the only real complaint is that most of the tracks have been heard before too many times; one or two more album tracks, or surprises, would have been welcome, to satisfy everybody. Yet you can't have it all, and for anybody who is in no way a collector, or even those too young to remember the originals, this is a fantastic introduction to one of the eighties true pop stars. (Web : google.co.uk) opposition de points de vue mais une forme de continuité argumentative, compatible 
avec l'intégration dans le co-texte droit de ce qui a été mentionné dans le co-texte gauche, qu'il s'agisse de nuancer le propos ou d'homogénéiser les représentations par la référence à une sorte d'énonciateur générique (quiconque, la sagesse populaire). C'est donc, là encore, l'absence de rupture, de disjonction, que marque still. pas nécessairement dans une relation d'opposition stricte, de type antonymique (même si on trouve aussi des énoncés comme (28)), mais en tout cas, des propriétés qui n'entretiennent pas de lien au niveau notionnel :

(28) Delores del Ruby appears from the reeds at Siwash Lake's edge, asleep yet awake. She has sunk so deep into the hole in her mind that gale and dust could not follow her.

Ainsi, un énoncé comme (29), dans lesquels les adjectifs coordonnés par yet renvoient à des propriétés qui sont notionnellement proches, est difficilement acceptable, dans la mesure où il semble suggérer que <be pretty> et <be graceful> devraient, a priori, s'exclure mutuellement :

(29) ? She was pretty yet graceful.

50 Ces phénomènes tendent à confirmer que l'on peut envisager yet comme un marqueur d'altérité, mettant en relation des éléments qui se trouvent, exceptionnellement, localisés dans une même situation (ici, incarnés dans un même référent). 


\section{Yet et still associés à une relation prédicative : We haven't finished yet/We're still working on it}

51 Il n'a pas encore été question de la valeur la plus représentée dans les corpus de ces marqueurs, leur valeur dite temporelle (ou aspectuelle), qui correspond aux cas dans lesquels ils portent sur une relation prédicative dont l'ancrage situationnel, la localisation temporelle, est en question. Comme il a été rappelé, les grammaires remarquent en général que still intervient dans des énoncés à polarité positive alors que yet n'apparaît que dans des contextes non-assertifs ou assertifs négatifs.

52 L'examen des corpus montre que la réalité est plus complexe et fait émerger un certain nombre de phénomènes qui sont, à notre connaissance, rarement observés. En premier lieu, la propension de still et de yet à la combinaison avec certaines formes aspectuelles mérite d'être mentionnée, même si, en définitive, elle ne fait que confirmer les hypothèses déjà formulées, selon lesquelles still serait un marqueur de continuité, et yet, un marqueur de discontinuité. Il n'est, en effet, guère surprenant de constater que still est plus fréquemment associé à $\mathrm{BE}+\mathrm{ING}$ que yet, y compris en contexte négatif.

(30) Yet when we visited Cabrini Greene almost a month after the accident, the elevator was still not working properly. (COCA)

(31) The old system had been removed, but the new ones were not yet working at full power. (BNC)

(32) The old mechanism already doesn't work. The new mechanism is not working yet. (COCA)

La position syntaxique des marqueurs, et les deux places différentes occupées par yet en (31) et (32) appellent un commentaire qui interviendra plus loin dans la démonstration.

Pour l'heure, rappelons que Culioli (1990, pp 91-114) opère une distinction entre deux types de négation, une négation dite primitive, qui correspond, très schématiquement, à une valuation négative émanant de l'énonciateur, et une négation construite, qui constitue une opération de prise en charge par l'énonciateur (une modalité d'assertion) d'une relation prédicative. C'est à ce deuxième type de négation que l'on a affaire dans des énoncés comme (30) à (32), dans lesquels celle-ci est marquée par not. Or, ce deuxième type de négation suppose, dans un premier temps, la construction de la relation prédicative, puis, le choix par l'énonciateur de l'assertion négative, à l'issue d'une pondération entre les polarités positive et négative.

Cette pondération opère de deux manières différentes avec still et yet. En effet, avec still, on constate que la relation prédicative affectée de la négation, c'est-à-dire l'événement < the elevator - not work> est toujours le cas, alors qu'on aurait pu s'attendre, compte tenu de la mise en place d'un nouveau repère temporel almost a month after the accident, à ce qu'on soit passé à un autre état de choses. Still indique que la relation prédicative reste dotée d'un repérage situationnel, malgré le changement de repère temporel. Rien n'est dit du pendant positif, <the elevator -work>, dont on ne sait à ce stade si l'événement qu'il dénote viendra à l'existence. Avec yet, on fait le constat que ce qui est le cas en situation, c'est l'événement correspondant à l'orientation négative de la relation <the new ones - not work at full power > ou <the new mechanism - not work>. Il n'en reste pas moins que, si la relation à polarité positive n'est pas dotée d'un ancrage situationnel, son existence même n'est pas remise en question. En (31) et (32), il ne fait nul doute que <the new ones - work at full power $>$ ou <the new mechanism - work> sera le cas à un moment donné. Nous y voyons une 
indication du fait qu'avec yet, la problématique de l'existence ou non de l'événement dénoté par la relation prédicative est dépassée. Il ne s'agit pas, à proprement parler, de repérer l'événement dans le temps, mais d'opposer ce qui est effectivement le cas, à ce qui devrait être le cas, ce à quoi on pourrait/devrait s'attendre.

D'ailleurs, les énoncés comme (31) et (32) dans lesquels la valeur attribuée à yet est d'ordre essentiellement temporel, sont assez rares. Très souvent, lorsque yet est associé à la forme aspectuelle BE + ING, on trouve des nuances telles celle émergeant dans l'énoncé (33), dans lequel BE + ING apparait dans son emploi dit de recatégorisation :

(33) She said,' What are you writing, Dad ?' He smiled at her.' I'm not really writing yet, just roughing out an idea. (BNC)

En effet, en (33), il est admis que le référent de Dad se trouve bien occupé à accomplir une tâche particulière, mais la nature de cette tâche fait débat. L'événement n'est, du point de vue du référent de Dad, pas une occurrence de /writing/ mais d'une autre notion /rough out an idea/.

La forme aspectuelle BE + ING localise le sujet d'emblée à l'Intérieur de la notion de procès, et n'est pas compatible avec une forme quelconque de discrétisation des occurrences, parce qu'elle ne permet ni l'altérité quantitative ni l'altérité qualitative. $C^{\prime}$ 'est donc sur un autre plan que va opérer yet, qui vient ici mettre en relation des représentations a priori contradictoires, des domaines notionnels distincts, comme on peut le voir en (33).

Comme nous l'avons fait remarquer, il apparaît difficile, lorsque l'on observe les corpus, de trouver des exemples dans lesquels yet, associé à BE+ING, aurait une valeur strictement et exclusivement temporelle, c'est-à-dire qu'il se contenterait de signaler que l'événement n'a pas encore eu lieu. Dans un exemple comme (34), par exemple, son emploi est justifié par la présence dans le co-texte gauche de 'You could give nanny a lift', qui présuppose la construction d'une représentation que l'on pourrait gloser par 'since you're leaving'. Dans ce cas, I'm not going yet ne vient pas seulement indiquer que la validation de la relation prédicative est différée, mais que son rattachement au moment présent constitue une représentation erronée.

(34) 'You could give nanny a lift.' 'I'm not going yet.' (BNC)

Si on supprimait yet, on obtiendrait (34'), dans lequel ce n'est pas le rattachement de l'événement au moment repère qui pose problème mais l'existence même de cet événement :

(34') 'You could give nanny a lift.' 'I'm not going.'

61 Selon les contextes, avec BE + ING, yet sera donc associé, soit à l'opposition entre deux domaines notionnels, par le biais d'un phénomène de recatégorisation (il se passe bien quelque chose, mais ce n'est pas ce que l'on croit, c'est d'un autre ordre), soit à l'opposition entre une valeur positive virtuelle et une valeur négative factuelle (la venue à l'existence de l'événement n'est pas en cause dans l'absolu, même si celui-ci ne possède pas d'ancrage temporel spécifique). Yet vient donc marquer un décalage entre ce qui est le cas et la représentation qu'on en a, ou ce que l'on prévoit de voir se réaliser. C'est toujours à une forme d'altérité que l'on a affaire, mais qui va porter soit sur le repère temporel (l'événement dénoté par la relation prédicative est le cas au moment repère, mais à un autre moment, c'est l'événement dénoté par la relation prédicative associé à une polarité positive qui est actualisé), soit sur la notion de procès (quelque chose est le cas, mais ça n'est pas ce que l'on imagine). 
62 Avec BE + ING, still indique la continuité d'un état de choses, et la forme aspectuelle permet le renvoi à un événement toujours en cours. Il s'agit d'un événement négatif en (30), mais on pourrait tout aussi bien avoir une polarité positive, comme en (35) :

(35) The woman I'm staying with comes from a mining family, and as I was watching the news I heard the ironing board creaking in the kitchen. She was still working, doing the ironing at ten o'clock at night. (BNC)

En (35), on observe, comme en (30), la localisation de l'événement dénoté par la relation prédicative par rapport à un nouveau repère temporel : at ten o'clock at night. On peut noter que ce genre de repère est absent des énoncés (31) et (32), ce qui montre bien que yet opère un travail différent et souligne l'opposition entre valeur positive et valeur négative bien davantage que l'attribution à l'une de ces valeurs d'une délimitation quantitative, d'un ancrage temporel spécifique. Yet vient donc exploiter et renforcer l'altérité préalablement construite par les autres termes de l'énoncé, par la forme non assertive ou assertive négative de la relation prédicative, qui suppose nécessairement une pondération entre les deux polarités et donc la construction, en amont, des valeurs positive et négative. Yet, on l'a dit, est la trace d'un repérage en altérité de l'occurrence sur laquelle il porte par rapport à une occurrence déjà construite dans l'énoncé, ce qui explique qu'il ne puisse apparaître dans des énoncés assertifs positifs que dans les contextes identifiés au début de cette étude, c'est-à-dire dans lesquels il ne porte pas sur le procès.

64 La comparaison de la combinaison de la forme HAVE + EN avec ces deux marqueurs met également au jour des phénomènes pertinents pour l'analyse. Ainsi, observons ce qui se passe en (36) et (37) :

(36) The product still has not reached the market and, while spokespersons for the Australian companies avoid saying that the project has been shelved, there appears to be no clear launch plan or marketing strategy. (BNC)

(37) Now clocking 186,000 miles per second. Now that's fast! Although, to be honest, we have to admit that the performance of the licensed cab has not reached light speed yet. (BNC)

65 La première remarque qui s'impose est d'ordre sémantique, alors que (36) signale le maintien d'une situation particulière, l'absence de changement, et donc la continuité, (37) implique que l'on envisage le passage d'un état de choses à un autre, même si dans ce nouvel état de chose, l'événement dénoté par la relation prédicative validée, n'existe pour le moment que sur le plan des représentations.

La seconde remarque concerne la construction linéaire de l'énoncé et la place du marqueur. Comme avec BE + ING, still est placé avant l'auxiliaire et ne peut occuper la position syntaxique de yet, et inversement. Still intervient à la jonction du sujet et du prédicat, déjà affecté, le cas échéant, de la négation. Tout se passe comme si, avec ce marqueur, il s'agissait de réaffirmer que la relation prédicative reste validée ou non validée dans un nouvel espace-temps. L'altérité, c'est-à-dire la possibilité que l'on soit passé à un autre état de choses, est prise en compte, puis éliminée.

D'ailleurs, il n'est pas souvent remarqué que still n'est pas compatible, quand il est associé à HAVE + EN, avec la forme assertive positive. En effet, lorsqu'on le rencontre dans cette configuration, il n'a plus une valeur temporelle mais concessive, comme en (38), alors que la valeur temporelle du marqueur est parfaitement compatible avec l'assertion négative :

(38) If in a few hours Bob starts swaying and keening and Jeannette is smoking feverishly, this still has been a good trip. / it has been a good trip nonetheless. (COCA) 
(39) The coroner still has not determined a cause of death. (COCA) esquelles on proposera des gloses distinctes :

(43) Not being in the present will contribute a great deal to the unhappiness caused by worries and anxieties of modern-day living, because we allow our minds to dwell upon the past which we can not change and the future which has not yet/even/ *for now happened. (BNC)

(44) The information is duff partly because the polls have not yet/even/ ? for now found a reliable way to deal with non-voters, or with Don't Knows (who may become Do Knows on election day). (BNC)

(45) Nobody was reported injured, but volcanologists said the eruption was only moderate.' We think the big one has not happened yet/* even/for now,' said Leonila Bautista. (BNC)

(46) I want to grow and develop as a person in areas that I have not explored yet/* even /for now. (BNC)

Les gloses proposées en (43) à (46) s'appuient sur des éléments de définition du marqueur yet fournis par le Concise Oxford English Dictionary. Leur acceptabilité est conditionnée par leur valeur sémantique, mais aussi par leur position syntaxique: for now est un circonstant de temps extraprédicatif, quant à even, il fait de la validation de la relation 
prédicative une condition minimale à la réalisation d'un but ultérieur, et on le trouve en position intraprédicative.

Il en résulte qu'en (45) et (46), yet va de pair avec la localisation de l'événement dans l'avenir (celui-ci ne possède pas d'ancrage temporel au moment repère, pourtant, son existence n'est pas remise en cause), la valeur négative correspondant à ce qui est effectivement le cas au moment repère: [...] the big one has not happened, I have not explored [the areas in which I want to grow and develop as a person. Dans le même temps, on envisage et projette dans le futur la validation de <the big one - happen $>$ et de $<I$-explore areas etc.>.

Si nous revenons à présent aux exemples (43) et (44), on voit que l'on se trouve dans un contexte dans lequel la validation de la relation prédicative <the future - happen $>$ et $<$ the polls - find a reliable way to deal with non-voters> constitue, en fait, un pré-requis à la validation de <the future - be changed $>$ et de <the information - be reliable>. Ces énoncés mettent en jeu des relations causales de type : pour avoir q, il faut p. Plus précisément, avec la négation, on a non q, puisque non $\mathrm{p}$. $P$ est posé comme condition nécessaire et minimale à $q$, c'est ce que fait apparaître la glose mettant en jeu le marqueur even. Dans la mesure où l'événement $p$, qui constitue la condition minimale, n'est pas le cas, $q$ ne peut venir à l'existence. Yet renforce l'opposition entre une valeur décrochée de l'actuel (si on avait $p$, on pourrait avoir $q$ ) et une valeur présentée comme étant réelle (comme on a non-p, on ne peut pas avoir q/on a forcément non-q). Autrement dit, yet permet de réactiver la valeur positive (si $p$ alors $q$ ) qui avait été, dans un premier temps, éliminée par l'énonciateur. En (44'), la suppression de yet ne permet pas de remobiliser la valeur positive. Celle-ci est d'abord prise en considération, puis évacuée via l'opération de négation dont not est la trace, par conséquent, <the information - be reliable> a été définitivement écarté et n'est pas présenté comme étant validable :

$\left(44^{\prime}\right)$ The information is duff partly because the polls have not found a reliable way to deal with non-voters, or with Don't Knows (who may become Do Knows on election day).

La différence entre les deux positions apparaît aussi en (47), dans lequel yet pourrait difficilement être déplacé en position postprédicative :

(47) The way she looked at them was odd, because there was no story here as yet, no drama whose next development or twist of plot or big scene we were all awaiting ; remember, they had not yet met or even looked at each other.

$\left(47^{\prime}\right)$ ? The way she looked at them was odd, because there was no story here as yet, no drama whose next development or twist of plot or big scene we were all awaiting ; remember, they had not met yet or even looked at each other.

Ici encore, la relation prédicative sur laquelle porte yet a une valeur essentiellement qualitative, dans la mesure où ce qui importe n'est pas qu'il existe à un moment donné une occurrence particulière de l'événement qu'elle dénote, mais plutôt le fait que cet événement constitue un prérequis notionnel à autre chose.

De même, dès lors que yet porte sur un prédicat intervenant au sein d'une hypothétique, la position intraprédicative entre l'auxiliaire et le verbe est la plus courante :

(48) You can't read them if they've not yet been written!

79 La position postprédicative, quant à elle, est associée à des propositions introduites par if dans des contextes dans lesquels la subordonnée n'est pas une hypothétique, mais une interrogative indirecte :

(49) I wonder if Ian's heard yet? 

l'opération de disjonction, la prise en compte d'une altérité irréductible entre l'occurrence sur laquelle il porte et le préconstruit, tandis que still peut être assimilé à l'opération de différenciation, par laquelle on va prendre en compte la possibilité de l'existence d'un état de choses potentiellement autre, pour, au bout du compte, éliminer systématiquement les différences entre les occurrences.

\section{BIBLIOGRAPHIE}

Charreyre, C. (2003). « Still, even so, all the same et "l'effet trampoline" ", paru dans Cycnos, Volume $23 \mathrm{n}^{\circ}$ 1, mis en ligne le 31 mai 2006, URL : http://revel.unice.fr/cycnos/index.html?id=302.

Culioli, A. (1990). Pour une linguistique de l'énonciation, tome 1, Opérations et représentations. Paris, Gap, Ophrys.

Culioli, A. (1999). Pour une linguistique de l'énoncation, tome 3, Domaine notionnel. Paris, Gap, Ophrys.

Morel, M-A. (1996). La concession en français. Paris, Gap, Ophrys. 
Quirk, R., Greenbaum, S., Leech, G., Svartik, J. (1985). A comprehensive grammar of the English language. New York, Longman.

Ranger, G. (1998). Les concessives en anglais, une approche énonciative. Paris, Gap, Ophrys.

Swan, M. (1980). Practical English Usage. Oxford, Oxford University Press.

Concise Oxford English Dictionary.

\section{RÉSUMÉS}

Cet article est une étude énonciative des marqueurs adverbiaux de l'anglais contemporain yet et still. A travers la variation des contextes syntaxiques et sémantiques (valeur temporelle, valeur concessive), il propose, par l'identification des contraintes associées à l'emploi de l'un et l'autre, de mettre au jour le principe qui sous-tend leur fonctionnement. Ainsi, l'étude propose de considérer que yet indique que le terme sur lequel il porte s'inscrit en disjonction par rapport à un préconstruit, alors que still est analysé comme la trace d'une continuité.

This paper consists in an utterer-centred approach to the functioning of the English adverbs yet and still. It aims at accounting for their modus operandi throughout the syntactic and semantic variations they are associated with, that is to say when they are used as time adjuncts or when they receive a concessive interpretation. Thus, this study suggests that it is possible to describe yet as marking disjunction between the term it introduces and what is preconstructed in the context. Still, on the other hand, is presented as involving continuity.

\section{INDEX}

Mots-clés : énonciation, adverbes, préconstruction, concession, temporalité

Keywords : enunciation, adverbs, preconstruction, concession, time markers

\section{AUTEUR}

\section{LISE HAMELIN}

Université de Cergy-Pontoise, LDI 